\title{
Lung squamous cell carcinoma associated with hypoparathyroidism with sensorineural deafness and renal dysplasia syndrome: a case report
}

This article was published in the following Dove Press journal:

OncoTargets and Therapy

\author{
Mariko Kojima' \\ Tatsuya Nagano' \\ Kyosuke Nakata' \\ Shigeo Hara ${ }^{2}$ \\ Naoko Katsurada' \\ Masatsugu Yamamoto' \\ Motoko Tachihara' \\ Hiroshi Kamiryo' \\ Kazuyuki Kobayashi' \\ Takeshi Usui ${ }^{3}$ \\ Yoshihiro Nishimura' \\ 'Division of Respiratory Medicine, \\ Department of Internal Medicine, \\ Kobe University Graduate School \\ of Medicine, Kobe, Hyogo, Japan; \\ ${ }^{2}$ Department of Diagnostic Pathology, \\ Kobe University Graduate School \\ of Medicine, Kobe, Hyogo, Japan; \\ ${ }^{3}$ Department of Medical Genetics, \\ Shizuoka General Hospital, Shizuoka \\ City, Shizuoka, Japan
}

Correspondence: Tatsuya Nagano Division of Respiratory Medicine, Department of Internal Medicine, Kobe University Graduate School of Medicine, 7-5-I Kusunoki-cho, Chuo-ku, Kobe, Hyogo 650-0017, Japan

$\mathrm{Tel}+8 \mathrm{I} 783825660$

Fax +8I $78382566 \mid$

Email tnagano@med.kobe-u.ac.jp

\begin{abstract}
Hypoparathyroidism with sensorineural deafness and renal dysplasia (HDR) syndrome is an autosomal dominant condition caused by mutations of the gene encoding the dual zinc-finger transcription factor, GATA3. A previous study identified some patients with GATA3 gene variants and breast cancer, suggesting that GATA3 variants may contribute to tumorigenesis in estrogen receptor 1-positive breast tumors; however, these patients did not have HDR syndrome. A 32-year-old nonsmoking Japanese woman was histologically diagnosed with lung squamous cell carcinoma associated with HDR syndrome and a c.C952T $>C$ (p.C318R) germline mutation in GATA3. This is the first report describing cancer in a patient with HDR syndrome. Our data indicates that GATA3 mutations may be a potential therapeutic target for lung cancer.
\end{abstract}

Keywords: lung cancer, GATA3, missense variant, hypoparathyroidism, sensorineural deafness, renal dysplasia

\section{Introduction}

Hypoparathyroidism with sensorineural deafness and renal dysplasia (HDR) syndrome (OMIM: 146255) is an autosomal dominant condition characterized by incomplete penetrance and clinical heterogneity, ${ }^{1}$ and is caused by mutations of GATA3 (OMIM: 131320). ${ }^{2}$ GATA3 consists of 6 exons which encode a dual zincfinger transcription factor. ${ }^{3}$ The C-terminal zinc finger of GATA3 is encoded by exon 5 and has a crucial role in DNA binding, whereas the N-terminal zinc finger is encoded by exon 4 and functions in stabilizing DNA binding and interactions with multitype zinc-finger proteins. ${ }^{4}$ Many types of GATA3 mutations can cause HDR syndrome, including intragenic deletions, along with nonsense, acceptor splice site, and missense mutations. ${ }^{3}$ The missense variants p.C318R and p.N320K are predicted to disrupt the C-terminal zinc finger of GATA3. ${ }^{3}$ The detailed structure of GATA3 has been described previously. ${ }^{3}$

Lung squamous cell carcinoma is a major histological subtype of non-small-cell lung cancer and is typically induced by cigarette smoking. ${ }^{5,6}$ Recently, potentially oncogenic mutations have been recognized in approximately $60 \%$ of lung adenocarcinoma, and molecular targeting therapies have been shown to improve the clinical outcomes of patients with these mutations. Nevertheless, oncogenic mutations are rare in lung squamous cell carcinoma, and the majority of patients are treated with conventional chemotherapy. ${ }^{5}$ Herein, we report a case of a young, nonsmoking woman with HDR syndrome who developed lung squamous cell carcinoma. 


\section{Reverse strand

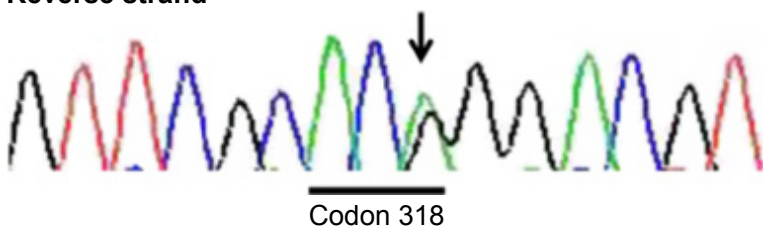

Figure I Polymerase chain reaction-direct sequencing.

Notes: Chromatogram showing the germline mutation in the case included in the present study. Arrow indicates the missense variant c.C952T $>C$ (p.C3I8R) in exon 5 of GATA3.

\section{Case report}

A 32-year-old nonsmoking Japanese woman was admitted to Kobe University Hospital in February 2017 to receive chemotherapy under hemodialysis.

The patient was congenitally disabled, with hearing difficulties. She underwent hemodialysis in 2012 because of congenital right renal deficiency and left renal hypoplasia. At that time, she had low levels of parathyroid hormone, due to hypoparathyroidism. After obtaining informed consent, she was diagnosed with HDR syndrome by analysis of a whole blood sample via polymerase chain reaction amplification of all GATA3 coding exons and exon-intron boundaries, followed by bidirectional Sanger sequencing. The results indicated that she carried the missense variant, c.C952T $>\mathrm{C}$ (p.C318R), in exon 5 of GATA3 (Figure 1). According to the standards and guidelines for the interpretation of sequence variants, this variant is classified as "likely pathogenic."7 Intriguingly, she was born to healthy parents and had no family history of a similar disorder (Figure 2).
In November 2016, she attended the hospital complaining of pain in the left back, and chest X-ray revealed left pleural effusion. She underwent a diagnostic bronchoscopy. Histopathological examination of tumor samples revealed dysplasia with coarse chromatin, and specimens stained positive for cytokeratin 5/6, and p63 (markers for squamous cell carcinoma), weakly positive for thyroid transcription factor-1, and negative for Napsin (markers for adenocarcinoma). Consequently, she was histologically diagnosed with lung squamous cell carcinoma (Figure 3). Her clinical stage of cancer progression was cT2aN0M1c, stage IVB, according to the TNM classification of the Union for International Cancer Control. Sequencing analysis of the tumor sample revealed no mutations of EGFR or EML4-ALK. Positron emission tomography-computed tomography revealed multiple bone metastases, including to the cervical, thoracic, and lumbar areas, along with the pelvis, humerus, and right ribs. Intriguingly, this is the only case of a young nonsmoking woman developing lung squamous cell carcinoma that has presented at Kobe University Hospital in the period 2011-2017 (Figure 4).

Upon hospitalization in February 2017, her Eastern Cooperative Oncology Group Performance Status was 2. A physical examination identified anemic palpebral conjunctiva and decreased left breath sounds. Chest X-ray revealed left pleural effusion, pleural thickening, and scoliosis (Figure 5). Chest computed tomography revealed mass-like opacity combined with atelectasis at the left apex, left pleural thickening, and left pleural effusion.

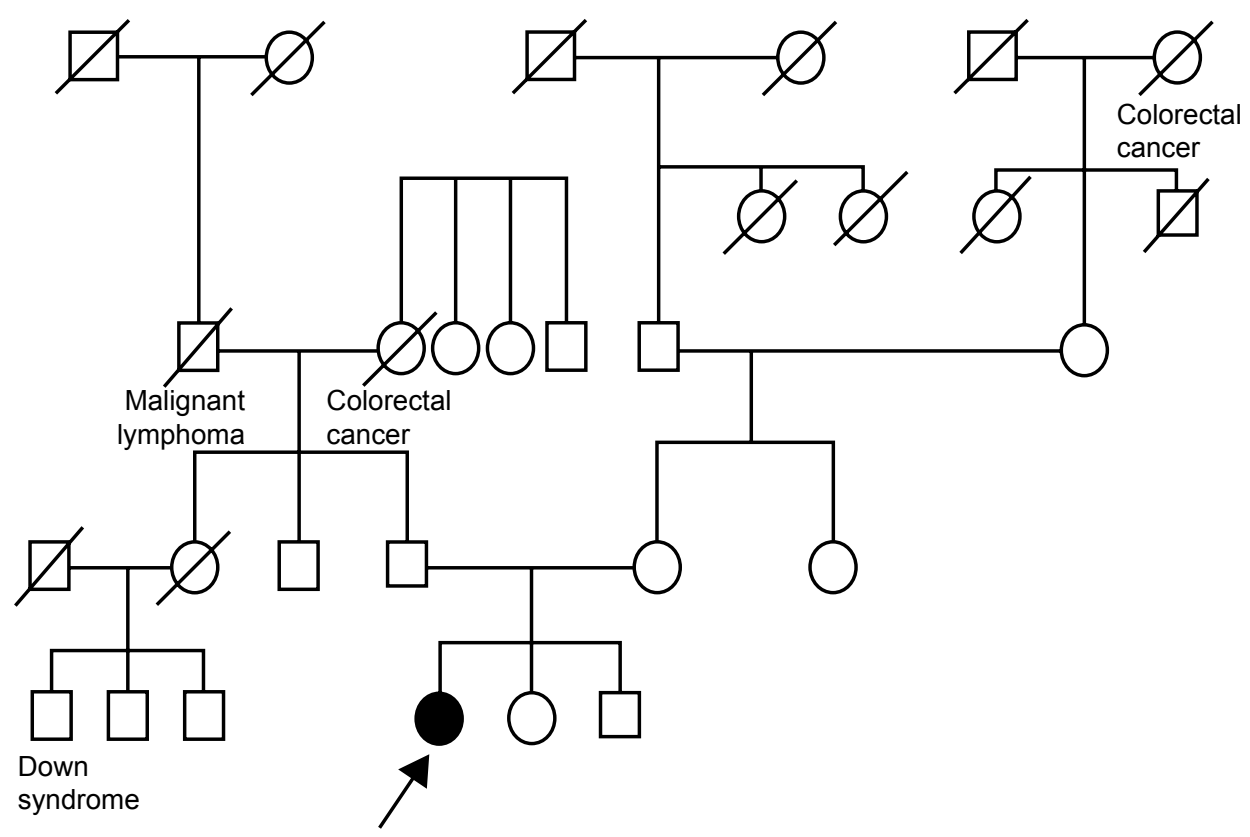

Figure 2 Pedigree.

Notes: Arrow indicates the proband. Males are represented by squares and females by circles. The filled circle indicates the affected female. A diagonal line through a square or circle indicates a deceased person. 


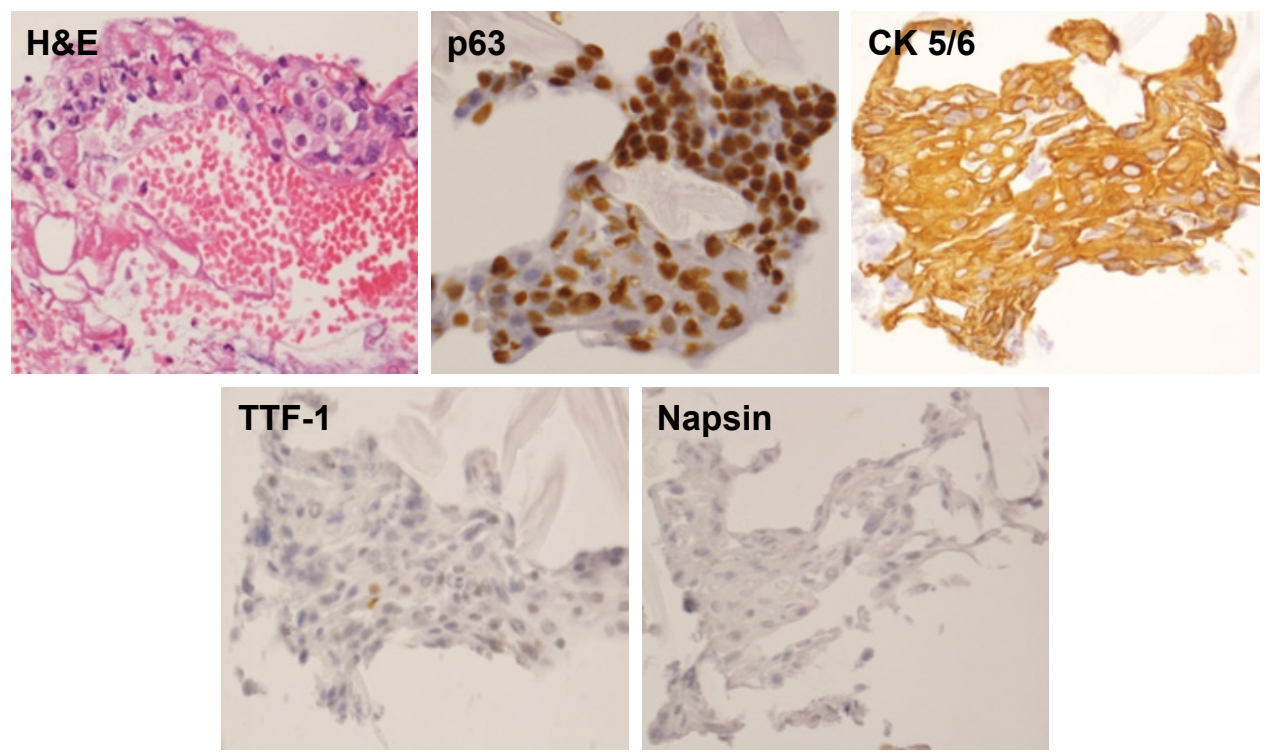

Figure 3 Images of bronchoscopic specimens stained for H\&E and the indicated markers.

Notes: Dysplasia with a coarse chromatin was observed in fibrous tissue. The tumor was positive for CK $5 / 6$ and p63, weakly positive for TTF-I, and negative for Napsin. Magnification is $\times 400$.

Abbreviations: CK 5/6, cytokerTIN 5/6; H\&E, hematoxylin and eosin; TTF-I, thyroid transcription factor-I.

She consented to a combination chemotherapy regimen to treat her non-small-cell lung cancer. On day 1 (nonhemodialysis day), she began treatment with carboplatin (area under the curve 5, estimated glomerular filtration rate $0,120 \mathrm{mg} /$ body $)$ and paclitaxel $\left(200 \mathrm{mg} / \mathrm{m}^{2}, 310\right.$ $\mathrm{mg}$ /body). On day 2, her serum AST and ALT levels transiently increased to 364 and $233 \mathrm{U} / \mathrm{L}$, respectively, before falling to Grade 3, according to the Common Terminology Criteria for Adverse Events version 4.0 (US National Cancer Institute, Rockville, MD, USA). From day 7 , she experienced appetite loss (Grade 2) and nausea (Grade 2). On day 9, she experienced febrile neutropenia

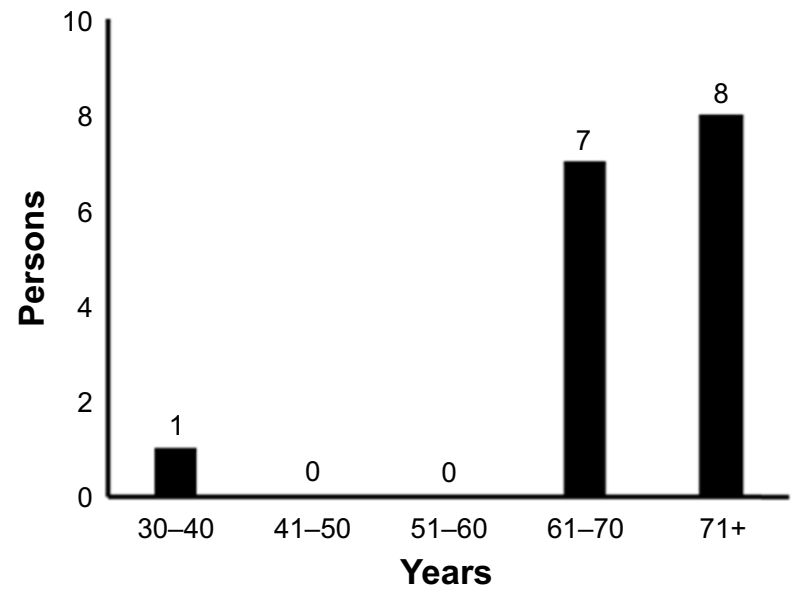

Figure 4 Age of nonsmoking patients diagnosed with lung squamous cell carcinoma at Kobe University Hospital from 2011 to 2017.

Note: Of 263 patients diagnosed with lung squamous cell carcinoma at Kobe University Hospital, 16 were nonsmokers. and was treated with $1 \mathrm{~g} / \mathrm{d}$ (hemodialysis day) or $2 \mathrm{~g} / \mathrm{d}$ (non-hemodialysis day) cefepime and $75 \mu \mathrm{g}$ of granulocyte colony-stimulating factor. Fortunately, she recovered from these toxicities and left Kobe University Hospital on day 17; however, the pain in her right upper arm and lower back were exacerbated (Numerical Rating Scale 5/10) from this point.

She was rehospitalized for chemotherapy in March 2017; however, her Eastern Cooperative Oncology Group Performance Status deteriorated to 3 because of pain induced by pathological fractures at sites of bone metastases (Th9, Th11, L2, and L4). Therefore, she was treated with vertebroplasty

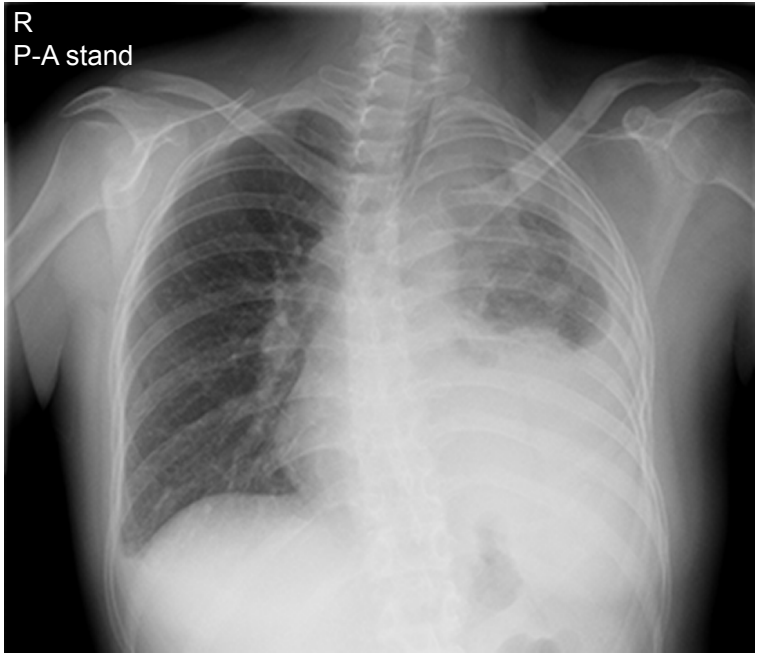

Figure 5 Chest X-ray.

Note: Left pleural effusion, pleural thickening, and scoliosis were detected. Abbreviations: R, right; P-A, posteroanterior. 
twice for the vertebral body metastases and radiation therapy for the bone metastases, rather than chemotherapy. Although her pain was improved to Numerical Rating Scale 3/10, she decided to terminate the chemotherapy and left Kobe University Hospital in March 2017.

\section{Discussion}

GATA3 is a member of a family of zinc-finger transcription factors present in vertebrate organisms and involved in the differentiation of breast epithelia, urothelia, and a subset of T lymphocytes. ${ }^{8}$ A previous study identified 5 patients with GATA3 gene mutations among 111 individuals with breast cancer; however, none of them had HDR syndrome. ${ }^{9}$ To our knowledge, there have been no reports describing patients with lung squamous cell carcinoma associated with HDR syndrome, although it has previously been suggested that disruption of GATA3 is associated with cancer.

Patients with HDR syndrome express various clinical phenotypes. Ferraris et $\mathrm{al}^{10}$ reported that of 77 patients, 48 $(62.3 \%)$ exhibited the complete clinical triad (ie, hypoparathyroidism, deafness, and renal dysplasia), 22 (28.6\%) lacked the renal disorder, 2 (2.6\%) did not exhibit hypoparathyroidism, and $5(6.5 \%)$ were not deaf. Although the pedigree of this patient indicates that no family members had symptoms associated with HDR syndrome, it is possible that some family members may have been partially or unknowingly affected. In addition, we were unable to determine the genetic status of other family members; therefore, we cannot conclude that the patient developed a de novo GATA3 p.C318R mutation. However, in this case, it is unlikely that there was undisclosed adoption, mistaken identity at the birthing facility, or marital infidelity. Indeed, Esch et al ${ }^{11}$ reported patients with a de novo $49 \mathrm{bp}$ frameshift deletion mutation in family 26/99, and Ferraris et al also reported a de novo heterozygous deletion of the nucleotides GG in codons 36 and 37 of GATA3. ${ }^{10}$

Lung adenocarcinoma accounts for the greatest proportion of lung cancers in women worldwide (ranging from $38 \%$ in the United States to $69 \%$ in Japan) ${ }^{12}$ however, lung squamous cell carcinoma accounts for only $11 \%$ of all lung cancers among women. ${ }^{12}$ Furthermore, in Japan, only $2.1 \%$ of lung cancer cases were diagnosed among women aged $0-34$ years. $^{13}$

The most significant risk factor for the development of lung cancer is tobacco smoking; smokers have a 15- to 30-fold higher risk of developing lung cancer than nonsmokers. ${ }^{14}$ Furthermore, exposure to indoor radon is considered to be the second-most significant environmental risk factor for lung cancer in the United States. ${ }^{15}$ Other risk factors for lung cancer include exposure to second-hand smoke; family history of lung cancer; air pollution; preexisting disease of the lungs, such as tuberculosis or pneumonia; exposure to a high dose of radiation; and exposure to industrial or chemical carcinogens, such as asbestos, silica, and arsenic. ${ }^{16-19}$ However, these risk factors were not applicable to this patient.

Given the above, we feel comfortable concluding that nonsmoking women in their thirties rarely develop cancer, except in response to genetic abnormalities. Indeed, recent studies have reported several genetic abnormalities that cause lung squamous cell carcinoma, including mutations in FGFR, PIL3CA, PTEN, AKT, DDR2, BRAF, PDGFRA, $S O X 2, E p h A 2$, and $I G F-1 R .^{20-22}$ However, these genetic abnormalities are relatively rare, and their precise frequencies are unknown.

GATA3 can regulate cell survival or terminal cell differentiation in many nontransformed tissues and also influence tumor differentiation and suppresses tumor dissemination in a luminal breast cancer model. ${ }^{23}$ In addition, GATA3 interacts with and stabilizes HIF-1 $\alpha$, which is important in the pathogenesis of various human cancers, and can enhance cancer cell invasiveness. ${ }^{24}$ These data support the hypothesis that the GATA3 mutation identified in the present case is associated with her development of lung squamous cell carcinoma.

Nakamura et al ${ }^{1}$ described another GATA3 missense variant in the same position (p.C318S; this patient carries p.C318R) without clinical history of cancer. Further investigations are needed to clarify the different effects of these changes on oncogenesis.

We analyzed a whole blood sample from the patient using polymerase chain reaction-direct sequencing and found that she was carrying the missense variant, p.C318R, in exon 5; however, we were unable to perform further analyses of genetic abnormalities, including of GATA3 in the lung tumor, because the patient refused to give consent.

\section{Conclusion}

This is the first report describing the co-occurrence of cancer in a patient with HDR syndrome. Our study may provide valuable information regarding the pathogenesis of lung squamous cell carcinoma and indicate that GATA3 mutations may be a potential therapeutic target for lung cancer.

\section{Acknowledgments}

We wish to thank the patient and family involved in this study for providing written informed consent for publication 
of this detailed case report. We also thank The Charlesworth Group for English language editing services.

\section{Author contributions}

MK, TN, and MT drafted the manuscript. KN, NK, and HK were involved in the clinical care of the patient. MY, KK, and YN interpreted clinical and laboratory data. SH provided histopathology results. TU coordinated genetic diagnosis, interpreted data, and critically revised the manuscript. All authors contributed toward data analysis, drafting and critically revising the paper and agree to be accountable for all aspects of the work. All authors read and approved the final manuscript.

\section{Disclosure}

The authors report no conflicts of interest in this work.

\section{References}

1. Nakamura A, Fujiwara F, Hasegawa Y, et al. Molecular analysis of the GATA3 gene in five Japanese patients with HDR syndrome. Endocr J. 2011;58(2):123-130.

2. Bilous RW, Murty G, Parkinson DB, et al. Brief report: autosomal dominant familial hypoparathyroidism, sensorineural deafness, and renal dysplasia. N Engl J Med. 1992;327(15):1069-1074.

3. Nesbit MA, Bowl MR, Harding B, et al. Characterization of GATA3 mutations in the hypoparathyroidism, deafness, and renal dysplasia (HDR) syndrome. J Biol Chem. 2004;279(21):22624-22634.

4. Tsang AP, Visvader JE, Turner CA, et al. FOG, a multitype zinc finger protein, acts as a cofactor for transcription factor GATA-1 in erythroid and megakaryocytic differentiation. Cell. 1997;90(1):109-119.

5. Tiseo M, Gelsomino F, Alfieri R, et al. FGFR as potential target in the treatment of squamous non small cell lung cancer. Cancer Treat Rev. 2015;41(6):527-539.

6. Sobue T, Yamamoto S, Hara M, Sasazuki S, Sasaki S, Tsugane S; JPHC Study Group. Japanese Public Health Center. Cigarette smoking and subsequent risk of lung cancer by histologic type in middle-aged Japanese men and women: the JPHC study. Int J Cancer. 2002;99(2): $245-251$.

7. Richards S, Aziz N, Bale S, et al. Standards and guidelines for the interpretation of sequence variants: a joint consensus recommendation of the American College of Medical Genetics and Genomics and the Association for Molecular Pathology. Genet Med. 2015;17(5):405-424.
8. Pandolfi PP, Roth ME, Karis A, et al. Targeted disruption of the GATA3 gene causes severe abnormalities in the nervous system and in fetal liver haematopoiesis. Nat Genet. 1995;11(1):40-44.

9. Usary J, Llaca V, Karaca G, et al. Mutation of GATA3 in human breast tumors. Oncogene. 2004;23(46):7669-7678.

10. Ferraris S, Del Monaco AG, Garelli E, et al. HDR syndrome: a novel "de novo" mutation in GATA3 gene. Am J Med Genet A. 2009;149A(4): 770-775.

11. Van Esch H, Groenen P, Nesbit MA, et al. GATA3 haplo-insufficiency causes human HDR syndrome. Nature. 2000;406(6794):419-422.

12. Youlden DR, Cramb SM, Baade PD. The international epidemiology of lung cancer: geographical distribution and secular trends. $J$ Thorac Oncol. 2008;3(8):819-831.

13. GLOBOCAN 2002. Cancer incidence, mortality and prevalence worldwide. IARC CancerBase No. 5 (version 2.0). Lyon, France: IARC Press; 2004. Available from: http://www-dep.iarc.fr/. Accessed June 29, 2017.

14. Sasco AJ, Secretan MB, Straif K. Tobacco smoking and cancer: a brief review of recent epidemiological evidence. Lung Cancer. 2004; 45(Suppl 2):S3-S9.

15. Alberg AJ, Ford JG, Samet JM; American College of Chest Physicians. Epidemiology of lung cancer: ACCP evidence-based clinical practice guidelines (2nd edition). Chest. 2007;132(Suppl 3):29S-55S.

16. Alberg AJ, Samet JM. Epidemiology of lung cancer. Chest. 2003; 123(Suppl 1):21S-49S.

17. Wakelee HA, Chang ET, Gomez SL, et al. Lung cancer incidence in never smokers. J Clin Oncol. 2007;25(5):472-478.

18. Bilello KS, Murin S, Matthay RA. Epidemiology, etiology, and prevention of lung cancer. Clin Chest Med. 2002;23(1):1-25.

19. Subramanian J, Govindan R. Lung cancer in never smokers: a review. J Clin Oncol. 2007;25(5):561-570.

20. Heist RS, Sequist LV, Engelman JA. Genetic changes in squamous cell lung cancer: a review. J Thorac Oncol. 2012;7(5):924-933.

21. Kim Y, Hammerman PS, Kim J, et al. Integrative and comparative genomic analysis of lung squamous cell carcinomas in East Asian patients. J Clin Oncol. 2014;32(2):121-128.

22. Bai Y, Kim JY, Watters JM, et al. Adaptive responses to dasatinibtreated lung squamous cell cancer cells harboring DDR2 mutations. Cancer Res. 2014;74(24):7217-7228.

23. Kouros-Mehr H, Bechis SK, Slorach EM, et al. GATA-3 links tumor differentiation and dissemination in a luminal breast cancer model. Cancer Cell. 2008;13(2):141-152.

24. Lin MC, Lin JJ, Hsu CL, Juan HF, Lou PJ, Huang MC. GATA3 interacts with and stabilizes HIF- $1 \alpha$ to enhance cancer cell invasiveness. Oncogene. 2017;36(30):4243-4252.
OncoTargets and Therapy

\section{Publish your work in this journal}

OncoTargets and Therapy is an international, peer-reviewed, open access journal focusing on the pathological basis of all cancers, potential targets for therapy and treatment protocols employed to improve the management of cancer patients. The journal also focuses on the impact of management programs and new therapeutic agents and protocols on

\section{Dovepress}

patient perspectives such as quality of life, adherence and satisfaction. The manuscript management system is completely online and includes a very quick and fair peer-review system, which is all easy to use. Visit http://www.dovepress.com/testimonials.php to read real quotes from published authors. 